\title{
Temperature Insensitive Current-Mode Four Quadrant Multiplier Using Single CFCTA
}

\author{
Sunti Tuntrakool , Peerawut Suwanjan and Winai Jaikla \\ Department of Engineering Education, Faculty of Industrial Education, King Mongkut's Institute of Technology Ladkrabang, Thailand
}

\begin{abstract}
A four quadrant multiplier of two current input signals using active building block, namely current follower cascaded transconductance amplifier (CFCTA) is presented in this paper. The proposed multiplier consists of only single CFCTA without the use of any passive element. The presented circuit has low impedance at current input node and high impedance at current output node which is convenient for cascading in current mode circuit without the need of current buffer circuits. The output current can multiply two input currents with temperature insensitivity. Moreover, the magnitude of output current can be controlled electronically via DC bias current. With only single active building block, the presented multiplier is suitable for integrated circuit implementation for analog signal processing. Simulation results from a PSpice program are presented in order to demonstrate the multiplier proposed here.
\end{abstract}

\section{Introduction}

Emerging of semiconductor technology has provided a new integrated circuit (IC) for developing the new topologies for both analog and digital circuits. Multipliers are circuits that are useful in the signal processing system [1]. Also, the multiplier can be found in many fields, for examples, modulation and de-modulation system, neural networks, adaptive filter etc. The design of analog circuits has been focused in the use of active building block, because it provides the flexibility for designer in the term of minimum number of external passive elements. Especially, the realization of circuit using electronically controllable active building blocks cab avoid the use of external resistor and can be easily controlled by microcontroller or microprocessor.

The synthesis of analog circuit based on current-mode technique has been gained significant attention, because current-mode circuit offers certain advantages such as greater linearity, simple structure, better accuracy, higherfrequency operation and larger dynamic range compared with op-amps-based circuits [2]-[3]. To make the current mode circuit cascading without the use of any additional buffer, the input impedance should be low and the output impedance should be high. Many current mode multipliers using active building block have been reported in the literature for example second generation current controlled current conveyor CCCII [4]-[6], operational transconductance amplifier (OTA) [7], current controlled current differencing transconductance amplifier (CCCDTA) [8], current controlled current differencing buffered amplifier (CCCDBA) [9].
The contribution of this paper is to propose a currentmode four quadrant multiplier using CFCTA as the active element. The output current is the multiplication of two input currents with temperature sensitivity. The proposed multiplier exhibits low input impedance for all two input nodes and high output impedance. Moreover, the magnitude of output current can be electronically controlled vial DC bias current. The proposed circuit consists of single CFCTA without any passive element. Through simulation using BJT CFCTA also demonstrates improvements in terms of performance.

\section{Principle of operation}

\subsection{Basic concept of Current Follower Cascaded Transconductance Amplifier (CFCTA)}

The active building block, namely current follower cascaded transconductance amplifier (CFCTA) is used to design the proposed multiplier. The briefly review of this device is explained here. Fig. 1 shows the electrical symbol and equivalent circuit of CFCTA. The input current port, namely $x$ port is low impedance. The output current $\mathrm{z}$ and $\mathrm{z}$-copy $\left(\mathrm{z}_{\mathrm{c}}\right)$ port are high impedance. Ideally, the output current $i_{z}$ and $i_{z c}$ is equal to input current $i_{x}$. The voltage at $\mathrm{z}$ port $\left(\mathrm{v}_{\mathrm{z}}\right)$ is converted to be the output current at $\mathrm{x}_{1}$ port through fist transconductance $\left(\mathrm{g}_{\mathrm{m} 1}\right)$ and the voltage at $\mathrm{x}_{1}$ port is converted to be output current at $\mathrm{x}_{2}$ port through second transconducatnce $\left(\mathrm{g}_{\mathrm{m} 2}\right)$. The impedance at port $\mathrm{x}_{1}$ and $\mathrm{x}_{2}$ is ideally high impedance. The transconductances $\mathrm{g}_{\mathrm{m} 1}$ and $\mathrm{g}_{\mathrm{m} 2}$ are electronically adjusted by DC bias current. The electrical characteristic of CFCTA is ideally written in equation (1) [10] 


$$
\left[\begin{array}{l}
V_{f} \\
I_{z, z_{c}} \\
I_{x_{1}} \\
I_{x_{2}}
\end{array}\right]=\left[\begin{array}{cccc}
0 & 0 & 0 & 0 \\
1 & 0 & 0 & 0 \\
0 & \pm g_{m 1} & 0 & 0 \\
0 & 0 & \pm g_{m 2} & 0
\end{array}\right]\left[\begin{array}{l}
I_{f} \\
V_{z} \\
V_{x 1} \\
V_{x 2}
\end{array}\right]
$$

The $\mathrm{g}_{\mathrm{m} 1}$ and $\mathrm{g}_{\mathrm{m} 2}$ are written, respectively, as

$$
g_{m 1}=\frac{I_{B 1}}{2 V_{T}} \text { and } g_{m 2}=\frac{I_{B 2}}{2 V_{T}}
$$

where $V_{T}$ is the thermal voltage. $I_{B 1}$ and $I_{B 2}$ are the DC bias current which can be only positive. Fig. 2 shows the internal construction of BJT CFTA.

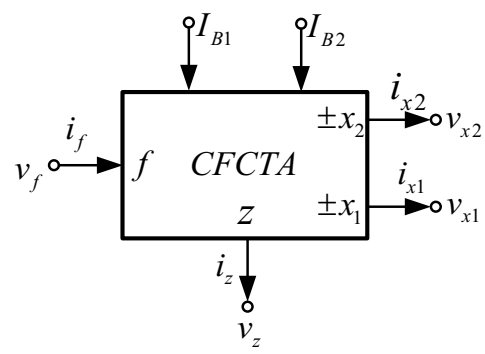

(a)

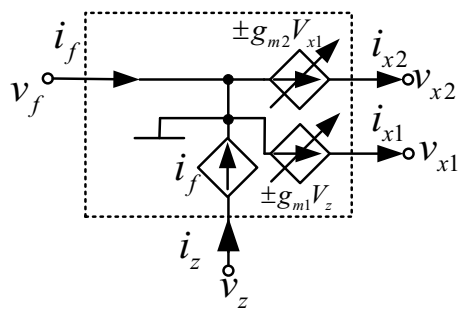

(b)

Figure 1. Circuit symbol and equivalent circuit of CFCTA.

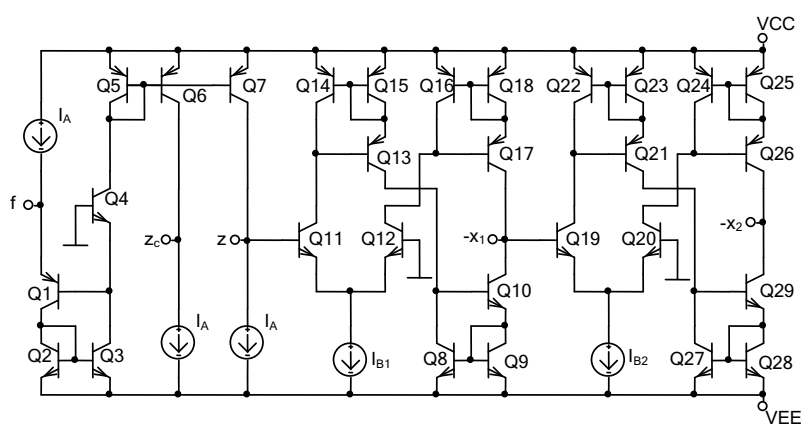

Figure 2. Internal construction of CFCTA [11]

\subsection{Temperature insensitive current-mode four quadrant multiplier}

Fig. 3 shows the proposed current-mode four quadrant multiplier. The proposed multiplier consists of only single CFCTA without the use of any passive element. It has low impedance at current input nodes $i_{\text {in1 }}$ and $i_{\text {in2 }}$. Also the output current node $i_{\text {out }}$ is high impedance which is ideal for cascade in current mode circuit without the use of buffer circuits. The DC current $I_{B}$ is biased to control the $g_{\mathrm{m} 1}$ and DC current $I_{B}$ adding with $i_{\text {in2 }}$ is biased to control $g_{m 2}$. So the $g_{m 1}$ and $g_{m 2}$ corresponding to bias current are respectively shown as

$$
g_{m 1}=\frac{I_{B}}{2 V_{T}}
$$

and

$$
g_{m 2}=\frac{I_{B}+i_{i n 2}}{2 V_{T}}
$$

It should be noted that $i_{\text {in2 }}$ can swing to be both positive and negative. However, the amplitude of $i_{\text {in2 }}$ should be lower or equal to $\mathrm{DC}$ bias current $\mathrm{I}_{\mathrm{B}}$. Considering the analysis of the circuit in Fig. 2, it yields the following output current

$$
i_{\text {out }}=i_{\text {in } 1}\left(1-\frac{g_{m 2}}{g_{m 1}}\right)
$$

Substituting Eq. (3) into Eq. (4), it yields

$$
i_{\text {out }}=-\frac{i_{\text {in1 }} i_{\text {in } 2}}{I_{B}}
$$

It should be noted from Eq. (6) that the output current $i_{\text {out }}$ is the multiplication of $i_{\text {in } 1}$ and $i_{\text {in } 2}$ and the division by $\mathrm{I}_{\mathrm{B}}$ with temperature insensitivity because the term of $V_{T}$ is not appeared in output current. Moreover, the proposed circuit is the four quadrant multiplier because $i_{\text {in } 1}$ and $i_{\text {in2 }}$ can swing to be both positive and negative direction. Also the amplitude of the output current can be controlled electronically by means of DC bias current $\mathrm{I}_{\mathrm{B}}$.

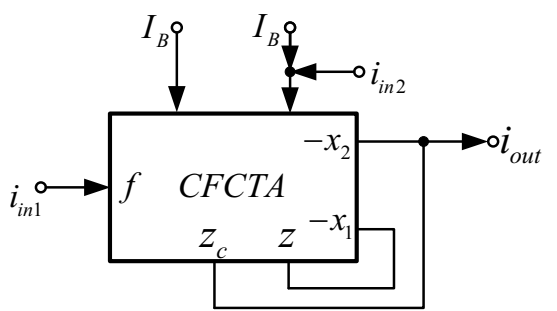

Figure 3. Proposed current mode four quadrant multiplier using single CFCTA

\section{Simulation results}

The proposed current multiplier was proved the performances by using the PSpice program. As illustrated in Fig. 2, the internal construction of CFCTA was constructed from BJT, the parameters of NPN and PNP transistors from the PR200N and NR200N bipolar transistors of ALA400 transistor array from AT\&T were used [12]. The proposed multiplier was biased with $\pm 2.5 \mathrm{~V}$ supply voltages. The first simulation result shown in Fig. 4 (a) is the DC transfer characteristic of the multiplier where $\mathrm{I}_{\mathrm{A}}=100 \mu \mathrm{A}, \mathrm{I}_{\mathrm{B} 1}=\mathrm{I}_{\mathrm{B} 2}=\mathrm{I}_{\mathrm{B}}=100 \mu \mathrm{A}$. $\mathrm{i}_{\text {in } 1}$ was sweep from $-120 \mu \mathrm{A}$ to $120 \mu \mathrm{A}$ and $\mathrm{i}_{\text {in2 }}$ was set in steps to $-100 \mu \mathrm{A}, 50 \mu \mathrm{A},-25 \mu \mathrm{A}, 0,25 \mu \mathrm{A}, 50 \mu \mathrm{A}$ and $100 \mu \mathrm{A}$. It is found that the input limitation of $\mathrm{i}_{\text {in1 }}$ is not higher than value of $\mathrm{DC}$ bias current $\mathrm{I}_{\mathrm{A}}$. The $\mathrm{DC}$ transfer characteristic is also illustrated in Fig. 4 (b) where $i_{\text {in2 }}$ was sweep from $-120 \mu \mathrm{A}$ to $120 \mu \mathrm{A}$ and $\mathrm{i}_{\text {in1 }}$ was set in steps to $-100 \mu \mathrm{A}, 50 \mu \mathrm{A},-25 \mu \mathrm{A}, 0,25 \mu \mathrm{A}, 50 \mu \mathrm{A}$ and 
$100 \mu \mathrm{A}$. The amplitude modulation is used as application for proposed multiplier. It is found that the input limitation of $\mathrm{i}_{\text {in2 }}$ is not higher than value of DC bias current $\mathrm{I}_{\mathrm{B}}$. The sinusoidal carrier signal with frequency of $10 \mathrm{kHz}$ was applied to $\mathrm{i}_{\text {in1 }}$ and the sinusoidal modulated signal with frequency of $1 \mathrm{kHz}$ was applied to $i_{\text {in2 } 2}$. The amplitude of both sinusoidal input signals were $160 \mu \mathrm{A}_{\mathrm{p}-\mathrm{p}}$. The results shown in figure 5 are the input signal and AM signal. Also, its output spectrum is shown in Fig. 6. The deviation of output current VS temperature variation is illustrated in Fig 7 where the temperature was sweep from $0-100{ }^{\circ} \mathrm{C}$. It is found that output deviation is about $3.68 \%$ to $6.93 \%$. This confirms the analysis in Eq. (6) that the output current slightly deviate correspondence to temperature variation. The output current for different value of $I_{B}$ is shown in Fig. 8. In this case, the $I_{B}$ was set to $100 \mathrm{~A}, 150 \mathrm{~A}$ and $200 \mathrm{~A}$. It is confirmed that the amplitude of output current can be electronically controlled by $I_{B}$ as shown in Eq. (6). The deviation of total harmonic distortions (THD) against amplitude of input currents $i_{\text {in } 1}$ and $i_{\text {in2 }}$ are illustrated in Fig. 9 and 10, respectively.

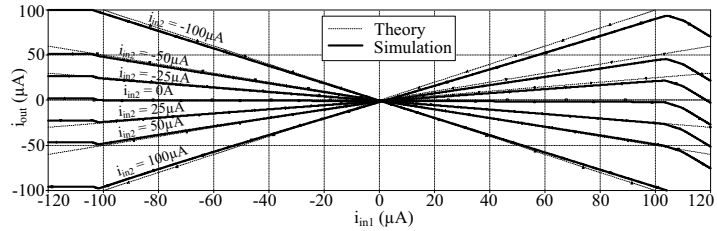

(a) $i_{\text {in1 }}$ sweep from $-120 \mu \mathrm{A}$ to $120 \mu \mathrm{A}$

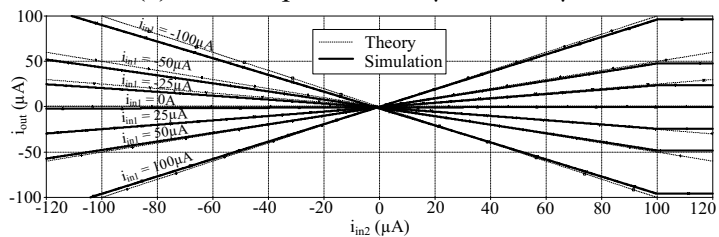

(b) $i_{\text {in2 } 2}$ sweep from $-120 \mu \mathrm{A}$ to $120 \mu \mathrm{A}$

Figure 4. DC transfer characteristic

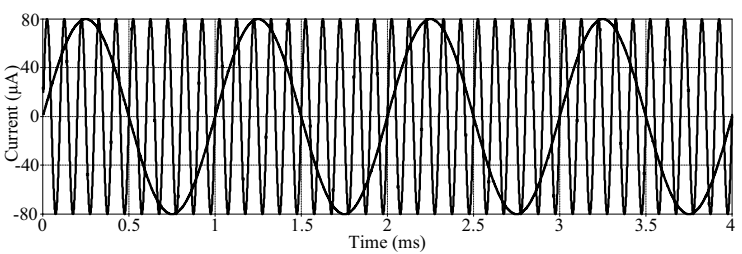

(a) Input currents of the multiplier

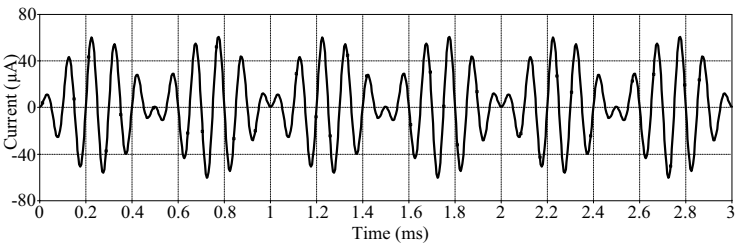

(b) AM output signal.

Figure 5. Application of proposed multiplier as amplitude modulation

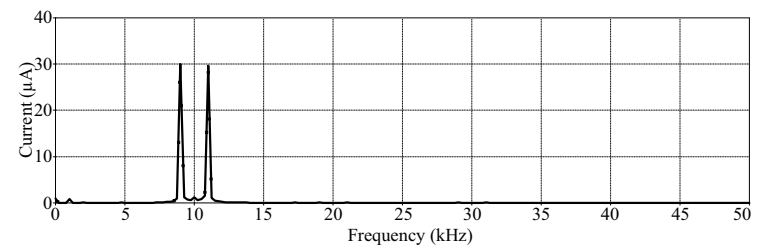

Figure 6. Output spectrum of Fig. 5 (a)

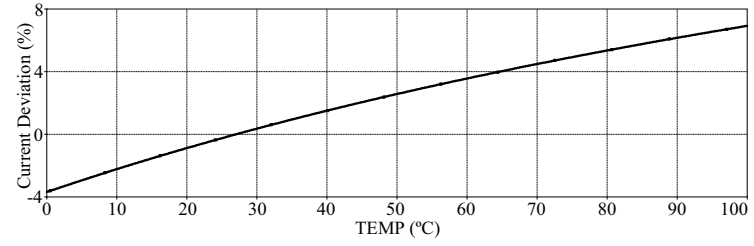

Figure 7. Output deviation VS temperature variation

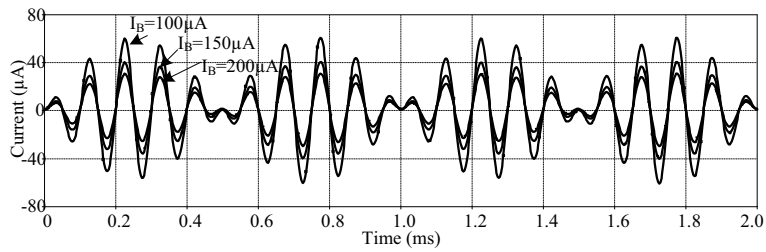

Figure 8. Output current for different value of $I_{B}$

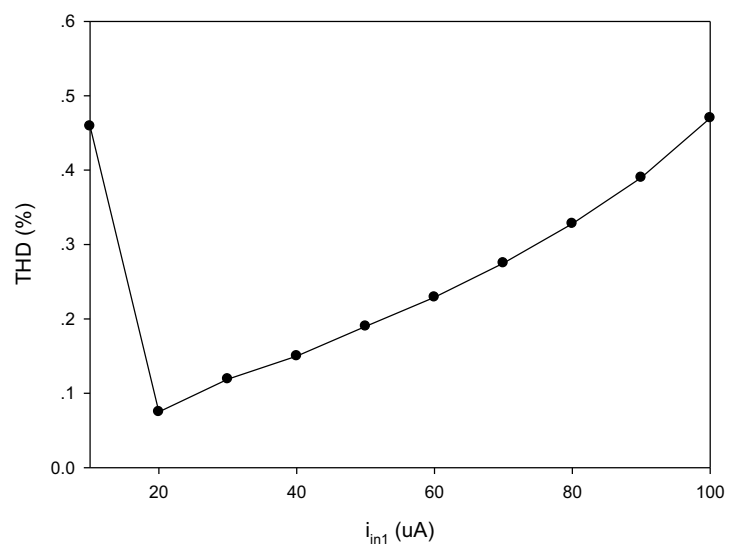

Figure 9. THD deviation against $i_{\text {in } 1}$ amplitude variation

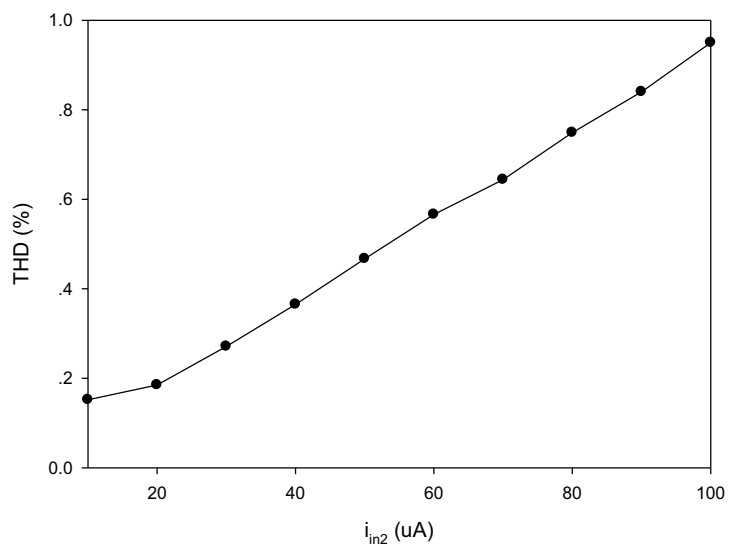

Figure 10. THD deviation against $i_{\text {in } 2}$ amplitude variation

\section{Conclusion}

This paper presents the current mode four quadrant multiplier. Single active building block used in this design is CFCTA. The output current is the multiplication of two input currents $i_{\text {ni1 }}$ and $i_{\text {in2 }}$ with temperature insensitivity. The output dynamic range depend on the DC bias current $\mathrm{I}_{\mathrm{A}}$ (in CFCTA) and $\mathrm{I}_{\mathrm{B}}$ (external bias current). The proposed multiplier doesn't require any passive element which is attractive for fabrication in chip. Moreover, the magnitude of the output current can be electronically adjusted via DC bias current $\mathrm{I}_{\mathrm{B}}$. The PSpice simulation results using BJT CFCTA based on 
parameters of PR200N and NR200N bipolar transistors of ALA400 agree well with theory expect.

\section{Acknowledgement}

Research described in this paper was financially supported from Faculty of Industrial Education, King Mongkut's Institute of Technology Ladkrabang (KMITL).

\section{References}

1. Ş.Ç. Yener, A. Uygur, H.H. Kuntman, "Ultra lowvoltage ultra low-power memristor based band-pass filter design and its application to EEG signal processing," Analog Integrated Circuits and Signal Processing, (2016), Article in press

2. E. Yuce, "Current-mode electronically tunable biquadratic filters consisting of only CCCIIs and grounded capacitors," Microelectronics Journal. 40, 719-1725, (2009).

3. G. Souliotis, "A current-mode automatic frequency tuning system for filters with current mirrors," International of Circuit Theory and Application. 38, 591-606, (2010)

4. M. T. Abuelma'atti and M. A. Al-Qahtani, "A current-mode currentcontrolled current-conveyorbased analogue multiplier/divider," International Journal of Electronics, 85, 71-77, (1998)

5. E. Yuce, "Design of a simple current-mode multiplier topology using a single CCCII+," IEEE Transactions on Instrumentation and Measurement. 57, $631-637,(2008)$
6. W. Petchakit, W. Kiranon, P. Wardkien, S. Petchakit, "A current-mode CCCII-based analog multiplier divider," International Conference on Electrical Engineering Electronics Computer Telecommunications and Information Technology (ECTI-CON), 221-224, (2010)

7. K. Kaewdang, C. Fongsamut, and W. Surakampontorn," Image processing using approximate datapath units," IEEE International Symposium on Circuits and Systems (ISCAS), 349352, (2003)

8. M. Siripruchyanun and W. Jaikla, W. "A currentmode analog multiplier/divider based on CCCDTA," International Journal of Electronics and Communications (AEU), 62, 223-227, (2008)

9. M. Siripruchyanun, and W. Jaikla "Current controlled current conveyor transconductance amplifier (CCCCTA): a building block for analog signal processing" Electrical Engineering, 90, 443453, (2008)

10. A. Chaichana and W. Jaikla, "Electronically tunable current-mode quadrature oscillator derived from first-oder allpass filter," 2014 European Modelling Symposium (EMS), 458 - 461, (2014)

11. W. Tangsrirat, "Novel current-mode and voltagemode universal filter using single CFTA," Indian Journal of Enineering \& Materials Sciences, 17, 99104, (2010)

12. D. R. Frey "Log-domain filtering: an approach to current-mode filtering," IEE Proc. Circuit Devices Syst., 140, 406-416, (1993). 